\title{
Modulating the Intentional Stance: Humanoid Robots, Narrative and Autistic Traits
}

\author{
Ziggy O’Reilly $^{1,2}$ (D), Davide Ghiglino ${ }^{1}$ (D), Nicolas Spatola ${ }^{1}$ (D), \\ and Agnieszka Wykowska ${ }^{1(\otimes)}$ \\ ${ }^{1}$ Social Cognition and Human-Robot Interaction (S4HRI), Istituto Italiano di Tecnologia, \\ Genova, Italy \\ Agnieszka.Wykowska@iit.it \\ 2 Department of Psychology, University of Turin, Turin, Italy
}

\begin{abstract}
To enhance collaboration between humans and robots it might be important to trigger towards humanoid robots, similar social cognitive mechanisms that are triggered towards humans, such as the adoption of the intentional stance (i.e., explaining an agents behavior with reference to mental states). This study aimed (1) to measure whether a film modulates participants' tendency to adopt the intentional stance toward a humanoid robot and; (2) to investigate whether autistic traits affects this adoption. We administered two subscales of the InStance Test (IST) (i.e. 'isolated robot' subscale and 'social robot' subscale) before and after participants watched a film depicting an interaction between a humanoid robot and a human. On the isolated robot subscale, individuals with low autistic traits were more likely to adopt the intentional stance towards a humanoid robot after they watched the film, but there was no effect on individuals with high autistic traits. On the social robot subscale (i.e. when the robot is interacting with a human) both individuals with low and high autistic traits decreased in their adoption of the intentional stance after they watched the film. This suggests that the content of the narrative and an individual's social cognitive abilities, affects the degree to which the intentional stance towards a humanoid robot is adopted.
\end{abstract}

Keywords: Intentional stance · Human-robot interaction · Autistic traits · Narrative

\section{Introduction}

\subsection{Social Cognition in Human-Robot Interaction}

Over the past decades researchers have been exploring the application of humanoid robots in a variety of settings, ranging from robot-assisted therapies for individuals on the autism spectrum [1,2], to tour guides in museums [3, 4]. However, the reception of robots in these environments is still a matter of debate. One of the ways to seamlessly integrate

Electronic supplementary material The online version of this chapter (https://doi.org/10.1007/ 978-3-030-90525-5_61) contains supplementary material, which is available to authorized users. 
robots in real-world (social) scenarios, could be to enhance their human-likeness, which in turn, might trigger social cognition mechanisms in the human counterpart [5]. If robots are able to trigger similar social cognitive mechanisms that are elicited during humanhuman interactions, they might also trigger a sense of connection and positively affect performance in collaborative tasks [5].

One social cognitive process which could be important to trigger, is the ascription of mental states towards an artificial agent [6]. In human-human interactions, this process of attributing mental states (such as beliefs, desires, intentions and emotions) to others, allows us to understand and predict behaviour [7,8]. The strategy of explaining behaviour with reference to mental states was termed by Daniel Dennett [9, 10] as adoption of the "Intentional Stance". Dennett argued that adopting the Intentional Stance towards humans, is the most efficient strategy to navigate social interactions. Thus, triggering the adoption of the Intentional Stance towards robots could enhance the ease to which individuals interact with robots in social tasks.

According to Dennett, the Intentional Stance is not always the most efficient strategy to explain the behaviour of all entities. For example, when individuals attempt to understand the behaviour of a robot, it could be more efficient to use knowledge about its functional design (i.e., 'the robot grabbed a glass because it was programmed to'), rather than describe the robot's behaviours based on mental states (i.e., 'the robot grabbed a glass because it was thirsty'). Dennett argued that when people use the former strategy they are adopting the Design Stance. Indeed, research has found variability in whether individuals describe the behaviour of a humanoid robot 'mechanistically' (i.e., adopt the Design Stance) or 'mentalistically' (i.e., adopt the Intentional Stance) [11-13]. However, it still remains to be answered what factors modulate whether individuals use mentalistic or mechanistic explanations for a humanoid robot's behaviour, or how to trigger participants to adopt the intentional stance towards a humanoid robot.

\subsection{Autistic Traits and Ascription of Mental States}

One factor which has been associated with an individual's ability to ascribe mental states in explaining behaviors of others, is the traits associated with autism spectrum disorder (ASD). ASD is a developmental disorder characterized by repetitive behaviors and difficulties in social communication and interaction [14]. Indeed, these social difficulties are thought to be caused by an impairment in the ability to reason about others with reference to mental states $[7,8]$. These difficulties are also present in neurotypical individuals with high levels of autistic traits, as measured by the Autism Quotient (AQ) test [15]. Indeed, Baron-Cohen and colleagues found that high AQ scores in a neurotypical population are associated with reduced abilities to infer the mental states of pictures of humans expressing emotions [16]. Interestingly, studies with children with ASD have found that impairments in ascription of mental states extend to humanoid robots [17, 18]. However, it is not known whether the low likelihood of ascribing mental states to humanoid robots is also present in neurotypical individuals with high autistic traits. Understanding the degree to which autistic traits modulate ascription of mental states towards robots, could help inform the design of humanoid robots to account for individual differences. 


\subsection{Using Narrative to Trigger Ascription of Mental States}

One of the ways to modulate how participants describe a robot's behaviour, could be by positioning it as a fictional character which communicates a narrative. Narrative has been defined as "a depiction of events driven by the intentional behaviour of one or more autonomous agents in a manner that manifests an imagined world which parallels the world of real experience," [19]. If a robot displays behaviour which appears intentional and drives a sequence of events, the most efficient strategy for the viewers to adopt to understand the narrative, should be to attribute goals, intentions and agency to the robot [20]. One way to investigate whether narrative can trigger individuals to describe the behaviour of a robot mentalistically, could be by using film. Indeed, a study using functional magnetic resonance imaging (fMRI), found that film footage activates brain regions involved in reasoning about others with reference to mental states [21]. Participants watched footage from the film Waking Life [22], which depicts two human characters physically embodied in the real world and the same footage converted into animated imagery (i.e., cartoonized). Brain regions involved in reasoning with reference to mental states were more active towards the 'real' film, in comparison to the cartoonized film. This suggests that film is more powerful when the characters are physically embodied in the real world, rather than cartoonized. Subsequently, showing participants a film where the events are driven by the behaviours of a humanoid robot, could enhance the likelihood that they explain a humanoid robot's behaviours mentalistically rather than mechanistically.

\subsection{Aims}

The question of whether narrative could trigger participants to adopt the intentional stance towards a humanoid robot and how this would vary based on the individual's level of autistic traits, has not yet been explored in detail in the literature to date. Therefore, the aim of this study was twofold: (1) to measure whether a film modulates participants' tendency to adopt the intentional stance toward a humanoid robot and; (2) to investigate whether autistic traits affect mentalistic attributions towards a humanoid robot. As stimuli, the study used a film titled While(Alive) \{\} [23] which displays the relationship between a humanoid robot and a human. The film is stop-motion animation and, as such, the characters are embodied in the physical-spatial world rather than in a virtual world. Thus, it is expected that the physical embodiment should enhance the likelihood of adopting the intentional stance towards a humanoid robot [21]. We also expected that While(Alive) \{\} would be more powerful at triggering adoption of intentional stance in individuals with low autistic traits, compared to individuals with high autistic traits based on previous research mentioned earlier $[15,17,18]$. 


\section{Methods}

\subsection{Participants}

For this study, 100 participants were recruited from an online platform Prolific (mean age $=27 ; S D=6 ; 50$ females, 1 other). The inclusion criteria for the study was (1) English as their first language and (2) consent. Additionally, half the participants had not reported a diagnosis of a developmental disability (i.e., neurotypical adults; $N=50$ ) and half reported a diagnosis of ASD $(N=50)$. However, since it was not possible to validate ASD diagnosis online, participants were split into groups based on their level of autistic traits rather than their reported diagnosis. Our experimental protocols followed the ethical standards laid down in the Declaration of Helsinki and were approved by the local Ethics Committee (Comitato Etico Regione Liguria).

\subsection{Stimuli}

A stop-motion animated film, While(Alive) \{\} was used as the experimental stimulus. While(Alive) \{\} depicts the evolution of a relationship between a robot and a human over the human's lifespan. The human ages overtime, dies and exits from the frame. In contrast, the robot does not undergo any physiological changes (see Fig. 1). There is no dialogue. The narrative is communicated predominately through hand gestures and music. The duration of the film is $1 \mathrm{~min}$ and $53 \mathrm{~s}$ (including title page, closing credits, and production card).

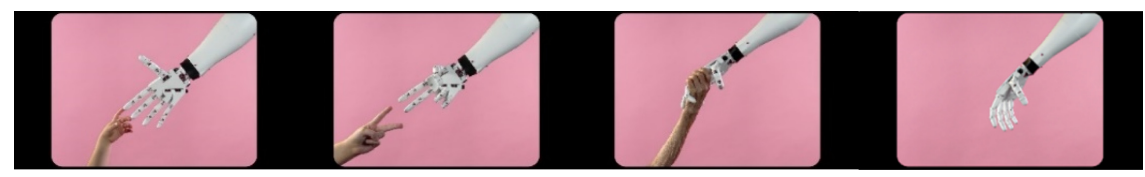

Fig. 1. Key frames from the stop-motion animated film While(Alive) \{\} . Full video available in [23]. (C) Cody Cameron-Brown and Ziggy O'Reilly.

\subsection{Measures}

Instance Test (IST). The IST contains sequences of images of the humanoid robot iCub interacting with objects and/or a human(s), designed to establish whether participants have a bias towards explaining iCub's behaviour mechanistically (i.e., Design Stance) or mentalistically (i.e., Intentional Stance) [13]. Underneath the sequence of images are two sentences representing possible explanations of the robot's behaviour. One of the sentences always explains iCub's behaviour mechanistically (i.e., 'iCub tracked the girls hand movements'), while the other always describes its behaviour mentalistically (i.e., 'iCub is interested in these objects'). These two sentences are located on the opposite poles of a bipolar scale, and participants are asked to move a slider toward the sentence that they think best suits the images. Participants instance scores (ISS) are calculated by converting the bipolar scale into a 0-100 scale for each item, which is then averaged 
across the items. The average indicates the participants preference for the mentalistic or mechanistic explanation, where 0 corresponds to the mechanistic explanation and 100 to the mentalistic explanation. The complete IST contains 34 items, and is associated with high internal consistency $(a=0.83,[24])$. This study used the shortened version developed by Spatola et al., [24] which contains 12 items, containing two subscales; 'isolated robot,' scale and the 'social robot,' scale (see Fig. 2). The IST was included to investigate whether While(Alive) \{\} modulated an individual's preference for mentalistic explanations of a humanoid robot's behaviour within-groups and between groups.
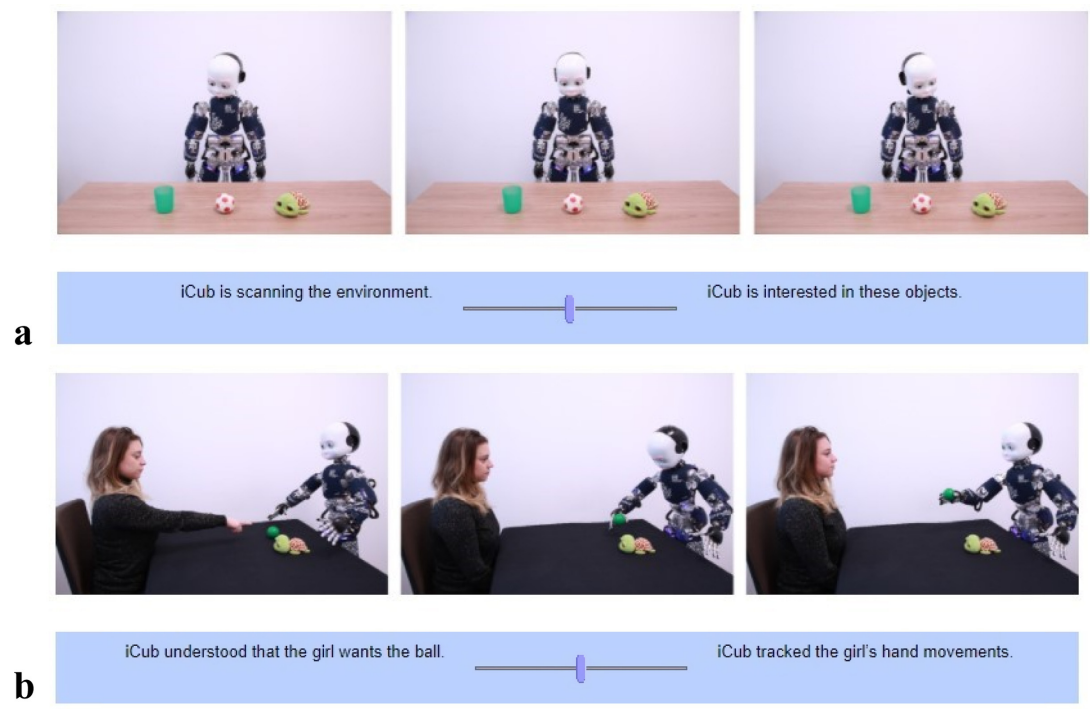

Fig. 2. Items from the InStance Test representative of the (a) isolated robot scale and (b) social robot scale.

\begin{abstract}
Abbreviated Adult Autism Spectrum Quotient (AQ-10). The AQ-10 is a short tenitem self-report questionnaire which measures the presence of autistic traits in individuals aged 16 years and older $[15,25]$. The 10 items (e.g., 'I find it difficult to work out people's intentions,' 'I find it easy to do more than one thing at once,' 'I often notice small sounds when others do not') are split equally into five subscales: 'attention to detail,' 'communication,' 'social,' 'attention switching,' and 'imagination,' which are answered on a four-point Likert scale marked by 'Definitely Agree', 'Slightly Agree', 'Slightly Disagree' and 'Definitely Disagree'. Any endorsements of autistic traits on positivelykeyed items (i.e., 'Definitely,' or 'Slightly Agree') are scored as 1, and disagreements are scored as 0 ; negatively key items are reverse scored. The total AQ scores range from 0 to 10. Allison, Auyeung and Baron-Cohen [25] found that the adult AQ-10 has high internal consistency $(\alpha=0.89)$ and high concurrent validity, compared to the full adult 50 -item version of the AQ $(r=0.92, p=.0001)$. The AQ-10 was included in the current study to split participants into two groups based on their level of autistic traits.
\end{abstract}




\subsection{Procedure}

All participants completed the online experiment in the same order. Before the main experiment begun, participants were asked to (1) check the display and audio of an unrelated short film and (2) answer demographic questions about English proficiency, age and sex. Then (after participants completed a practice item from the IST), they were presented with the first half of the IST in a pseudo-randomized order. Next, participants watched While(Alive) \{\} on full screen with headphones. After watching While(Alive) \{\} , they completed the second half of the IST, which was presented in a pseudo-randomized order and completed the AQ-10. Finally, as a quality control check, participants were asked "did you put reasonable effort into this study?" The study took approximately 20 min to complete.

\subsection{Analysis}

For each group, the ISS was screened for outliers using the 3.0 inter-quartile range rule [26]. Based on an a priori hypothesis, a two-way repeated measures ANOVA was conducted per group to investigate whether the subscale (social robot vs. isolated) effected the ISS before and after participants watched While(Alive) \{\}. Additional analyses using paired samples t-tests were conducted to make post hoc comparisons between the social robot subscale and the isolated robot subscale, for each group. The data was normally distributed according to the Shapiro-Wilk test of normality. We applied a Bonferroni correction. All analyses were performed using SPSS statistical package version 24 (SPSS Inc, Chicago, IL).

\section{Results}

\subsection{Data Screening}

Eight participants were excluded for the analysis because they reported that their English was less than excellent. An additional participant was excluded because their English was less than excellent and they answered "no" to the question "did you put reasonable effort into this study?" Four more participants were excluded because their mean time to respond to the IST was above the 3.0 interquartile range rule [26]. Finally, 14 participants were excluded because they took $40 \mathrm{~s}$ or less to respond to 6 items the IST. This yielded a final sample of 73 participants (see Table 1). The participants were split into a low AQ group and a high $\mathrm{AQ}$ group based on median $\mathrm{AQ}$ scores $(M d n=6)$. The $\mathrm{AQ}$ scores for the high AQ group were significantly greater than for the low AQ group; $(t(71)=-15.097$, $p=.000)$. No outliers were detected. 
Table 1. Descriptive Statistics for the Low AQ group and the High AQ group.

\begin{tabular}{l|l|l}
\hline & Low AQ & High AQ \\
\hline$N$ (male:female:other) & $36(12: 24: 0)$ & $37(22: 14: 1)$ \\
\hline Age & $27(7)$ & $27(6)$ \\
\hline AQ-10** & $3.28(1.34)$ & $7.67(1.19)$ \\
\hline
\end{tabular}

Note. Values are given as mean (SD) unless otherwise stated. Asterisks indicate when groups significantly differ from each other. $* * p<0.001$

\subsection{Main Analysis}

Low AQ Group. According to a two-way repeated measures ANOVA, there was a significant main effect of IST subscale (isolated robot vs. social robot; $F(1,35)=12.13$, $p=.001, \eta_{\mathrm{p}}{ }^{2}=.257$; see Fig. $\left.3 \mathrm{a}\right)$. The marginal mean for the social robot subscale was higher $(M=49.30)$ than for the isolated robot subscale $(M=38.35)$. There was no significant main effect of time (i.e., before or after participants watched While(Alive) \{\} ) $\left(F(1,35)=.569, p=.456, \eta_{\mathrm{p}}{ }^{2}=.016\right)$. However, there was a significant interaction between pre-/post- changes and the IST subscales $\left(F(1,35)=18.95, p<.001, \eta_{\mathrm{p}}{ }^{2}=\right.$ .351; see Fig. 3a). For the isolated robot subscale of the IST, separate comparisons showed that ISS differed significantly before and after watching While(Alive) \{\} ( $t(35)$ $=-3.064, p=.004)$. The ISS after watching While(Alive) \{\} was higher $(M=46.06, S D$ $=20.92)$ than before $(M=30.64, S D=25.43)$. For the social robot subscale of the IST, we also found a significant difference between the ISS before and after watching While(Alive $)\{\}(t(35)=3.088, p=.004)$. However, for this subscale, the ISS after watching While(Alive) \{\} was lower $(M=43.93, S D=22.39)$ than before $(M=54.67$, $S D=21.09)$.

High AQ Group. According to a two-way repeated measures ANOVA, there was a significant main effect of IST subscale $\left(F(1,36)=14.925, p<.001, \eta_{\mathrm{p}}{ }^{2}=.293\right.$; see Fig. 3b). The marginal mean for the social robot subscale was higher $(M=46.48)$ than for the isolated robot subscale $(M=34.96)$. There was no significant main effect of time (i.e., before or after participants watched While(Alive \{\}$)(F(1,36)=3.089, p=$ $\left..088, \eta_{\mathrm{p}}{ }^{2}=.079\right)$. However, also for this group, there was also a significant interaction between pre-/post- changes and the subscale $\left(F(1,36)=6.66, p=.014, \eta_{\mathrm{p}}{ }^{2}=.156\right.$; see Fig. $3 b)$. For the isolated robot subscale, there was no significant difference between the ISS before and after watching While(Alive) \{\}$(t(36)=.63, p=.950)$. In other words, there were no changes before $(M=35.10, S D=25.08)$ or after $(M=34.82, S D=$ 21.06) watching While(Alive) \{\} for this subscale of IST. On the contrary, for the social robot subscale of IST, there was also a significant difference between the ISS before and after watching While(Alive $)\{\}(t(36)=2.673, p=.011)$. The ISS after watching While(Alive) \{\} was lower $(M=39.71, S D=26.28)$ than before $(M=53.24, S D=$ 26.10), paralleling the pattern for low-AQ group of participants. 

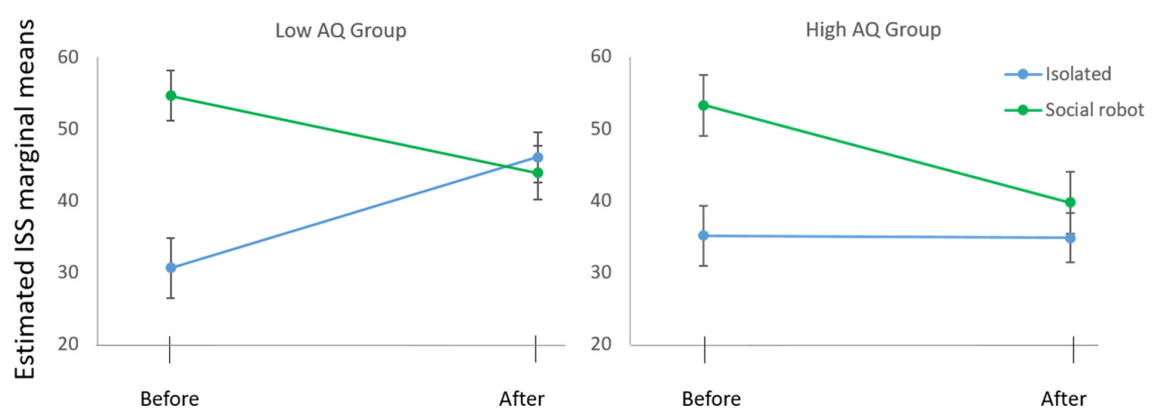

Fig. 3. (a) Interaction effect across subscales before and after watching While(Alive) \{\} for the low AQ group. (b) Interaction effect across subscales before and after watching While(Alive) \{\} for the high AQ group.

\section{Discussion}

The primary aim of the present study was to investigate whether a narrative of an interaction between a human and a humanoid robot could modulate the adoption of the intentional stance towards a humanoid robot. Secondly, it aimed to investigate whether the degree of autistic traits affected the modulation. Adoption of the Intentional Stance was measured by administering the InStance Test (IST) before and after participants watched a film titled While(Alive) \{\} . The IST contained two subscales; the isolated robot scale, which shows the humanoid robot iCub interacting with objects, and the social robot scale, which depicts iCub interacting with a human(s).

Our results showed that for both groups of participants, the Intentional Stance score decreased on the IST social robot subscale (robot depicted in the presence of a human) after watching While(Alive)\{\}. This could be explained by the narrative underlying the interaction between the human and the robot in the film. Majority of the human-robot interaction is driven by the mental states of the human, rather than of the robot. For example, in the beginning of the film, the infant's curiosity propels her to touch the robot. As such, the human character is more 'active,' in comparison to the more 'passive' robot character. Therefore, it is possible that to understand the narrative, it is sufficient to attribute mental states towards the 'active character,' (i.e., the human), rather than the 'passive character,' (i.e., the robot). Subsequently, While(Alive) \{\} may trigger participants to perceive the behaviour of the robot mechanistically, by contrast to the mentalistic behaviour of the human. The decrease in scores in the social robot scale (i.e., the shift towards more mechanistic explanations) could reflect this.

Interestingly, on the isolated robot subscale of the IST, the very same video clip increased the IST score, but only for the low-AQ group of participants. This suggests that individuals with lower degree of autistic traits might show higher cognitive flexibility [27] and might be more likely to attribute human-like traits (such as intentionality) to non-human agents (such as humanoid robots), especially, after watching an emotionally evocative movie depicting human-robot interaction. Interestingly, although the narrative of the movie highlighted the human as the intentional agent, thereby, increasing - by contrast - the mechanistic perception of a robot when in interaction with a human (in the social robot subscale), the human intentionality was transferred to the robot, when the 
robot was displayed alone in the IST, being the sole agent in the scenario. This, however, occurred only among participants with higher social aptitude (lower AQ traits).

Overall, our results suggest that adoption of intentional stance is fluid, and can be modulated by various factors, such as a background narrative induced by a movie or by a social context in which a robot is presented. Importantly, the way these factors influence adoption of the Intentional Stance depends also on individual differences in social aptitude, as manifested by the degree of individual AQ traits. However, due to participants ability to use Prolific, our results are presumably based on a sample of individuals with high cognitive functioning. As such, the results should not be interpreted as representative of all individuals on the autism spectrum. Nevertheless, knowledge about individual AQ traits should be considered in the design of social robots.

Acknowledgements. This work has received support from the European Research Council under the European Union's Horizon 2020 research and innovation programme, ERC Starting grant ERC-2016-StG-715058, awarded to Agnieszka Wykowska. The content of this paper is the sole responsibility of the authors. The European Commission or its services cannot be held responsible for any use that may be made of the information it contains. We would also like to thank Serena Marchesi for assisting with creating the online questionnaire.

\section{References}

1. Silvera-Tawil, D., Roberts-Yates, C.: Socially-assistive robots to enhance learning for secondary students with intellectual disabilities and autism. In: 27th IEEE International Symposium on Robot and Human Interactive Communication (RO-MAN) 2018, pp. 838-843. IEEE (2018)

2. Ricks, D.J., Colton, M.B.: Trends and considerations in robot-assisted autism therapy. In: IEEE International Conference on Robotics and Automation 2010, pp. 4354-4359. IEEE (2010)

3. Burgard, W., et al.: Experiences with an interactive museum tour-guide robot. Artif. Intell. 114(1-2), 3-55 (1999)

4. Nourbakhsh, I., et al.: The personal exploration rover: educational assessment of a robotic exhibit for informal learning venues. Int. J. Eng. Educ. 22(4), 777 (2006)

5. Wiese, E., Metta, G., Wykowska, A.: Robots as intentional agents: using neuroscientific methods to make robots appear more social. Front. Psychol. 8, 1663 (2017)

6. Gray, H.M., Gray, K., Wegner, D.M.: Dimensions of mind perception. Science 315(5812), 619 (2007)

7. Baron-Cohen, S., Leslie, A.M., Frith, U.: Does the autistic child have a "theory of mind"? Cognition 21(1), 37-46 (1985)

8. Baron-Cohen, S.: Theory of mind and autism: a fifteen year review. Underst. other Minds: Perspect. Dev. Cogn. Neurosci. 2, 3-20 (2000)

9. Dennett, D.C.: Intentional systems. J. Philos. 68(4), 87-106 (1971)

10. Dennett, D.C., Haugeland, J.: Intentionality. In: The Oxford Companion to the Mind. Oxford University Press (1987)

11. Bossi, F., Willemse, C., Cavazza, J., Marchesi, S., Murino, V., Wykowska, A.: The human brain reveals resting-state activity patterns that are predictive of biases in attitudes toward robots. Sci. Robot. 5(46), 1-8 (2020)

12. Salichs, M.A., et al. (eds.): ICSR 2019. LNCS (LNAI), vol. 11876. Springer, Cham (2019). https://doi.org/10.1007/978-3-030-35888-4 
13. Marchesi, S., Ghiglino, D., Ciardo, F., Perez-Osorio, J., Baykara, E., Wykowska, A.: Do we adopt the Intentional Stance toward humanoid robots? Front. Psychol. 10, 450 (2019)

14. American Psychiatric Association.: Diagnostic and statistical manual of mental disorders (DSM-5®): American Psychiatric Pub. (2013)

15. Baron-Cohen, S., Wheelwright, S., Skinner, R., Martin, J., Clubley, E.: The autism-spectrum quotient (AQ): Evidence from asperger syndrome/high-functioning autism, males and females, scientists and mathematicians. J. Autism Dev. Disord. 31(1), 5-17 (2001)

16. Baron-Cohen, S., Wheelwright, S., Hill, J., Raste, Y., Plumb, I.: The, "Reading the Mind in the Eyes" test revised version: a study with normal adults, and adults with Asperger syndrome or high-functioning autism. J. Child Psychol. Psychiatry 42(2), 241-251 (2001)

17. O'Reilly, Z., Silvera-Tawil, D., Tan, D.W., Zurr, I.: Validation of a novel theory of mind measurement tool: the social robot video task. In: Companion of the 2021 ACM/IEEE International Conference on Human-Robot Interaction, pp. 89-93 (2021)

18. Zhang, Y., et al.: Theory of robot mind: false belief attribution to social robots in children with and without autism. Front. Psychol. 10, 1732 (2019)

19. Mar, R.A.: The neuropsychology of narrative: Story comprehension, story production and their interrelation. Neuropsychologia 42(10), 1414-1434 (2004)

20. Frith, C.D., Frith, U.: Interacting minds-a biological basis. Science 286(5445), 1692-1695 (1999)

21. Mar, R.A., Macrae, C.N.: Triggering the intentional stance. In: Novartis Foundation Symposium, vol. 278, p. 111. John Wiley, Chichester (2006)

22. Linklater, R.: Waking Life. Fox Searchlight Pictures, Los Angeles (2001)

23. Cameron-Brown, C., O'Reilly, Z.: "While(Alive)\{\}." YouTube Video. https://www.youtube. $\mathrm{com} /$ watch?v=qeCmqKiueu8

24. Spatola, N., Marchesi, S., Wykowska, A.: The Instance Task: how to measure the mentalistic bias in human-robot interaction, 28 May 2021.https://doi.org/10.31234/osf.io/b3wtq

25. Allison, C., Auyeung, B., Baron-Cohen, S.: Toward brief "red flags" for autism screening: the short autism spectrum quotient and the short quantitative checklist in 1,000 cases and 3,000 controls. J. Am. Acad. Child Adolesc. Psychiatry 51(2), 202-212 (2012)

26. Hoaglin, D.C., Iglewicz, B.: Fine-tuning some resistant rules for outlier labeling. J. Am. Stat. Assoc. 82(400), 1147-1149 (1987)

27. Wykowska, A.: Social robots to test flexibility of human social cognition. Int. J. Soc. Robot. 12, 1203-1211 (2020) 